TITLE:

\title{
Accessibility and size of Valonia cellulose microfibril studied by combined deuteration / rehydrogenation and FTIR technique
}

\section{$\operatorname{AUTHOR}(\mathrm{S})$ :}

Horikawa, Yoshiki

\section{CITATION:}

Horikawa, Yoshiki. Accessibility and size of Valonia cellulose microfibril studied by combined deuteration /

rehydrogenation and FTIR technique. Sustainable humanosphere : bulletin of Research Institute for Sustainable

Humanosphere Kyoto University 2008, 4: 15-16

ISSUE DATE:

2008-09-01

URL:

http://hdl.handle.net/2433/182116

RIGHT: 


\title{
Accessibility and size of Valonia cellulose microfibril studied by combined deuteration/rehydrogenation and FTIR technique
}

\author{
Yoshiki Horikawa \\ (Laboratory of Biomass Morphogenesis and Information) \\ RISH, Kyoto University
}

\begin{abstract}
Deuterium has been used as a chemical reagent to estimate the accessibility of cellulose because in organic macromolecules such as cellulose microfibrils, deuterium can replace the hydrogen molecule that comprises the hydrogen bond. In the studies reported in early literature, cellulose specimens were soaked in heavy water $\left(\mathrm{D}_{2} \mathrm{O}\right)$, and crystallinity was estimated in terms of structural accessibility by calculating the difference in weight (Frilette et al. 1948). With the advent of infrared spectroscopy, accessibility could be estimated by deuteration. Cellulose samples were immersed in $\mathrm{NaOD}$ solution for deuteration, washed in $\mathrm{D}_{2} \mathrm{O}$, and dried in a box containing dry nitrogen. Deuterium can be used for infrared spectroscopy because the mass difference due to hydrogen dramatically affects the frequency of molecular vibrations. In cellulose, OD bond vibrations are observed in locations free of other signals. The initial infrared spectrum immediately after exposure of the deuterated specimens to air showed strong OD bands. The intensity of these bands decreased with time due to moisture in the air.
\end{abstract}

Recently, by high-temperature annealing of the sample at $260^{\circ} \mathrm{C}$ in $0.1 \mathrm{~N} \mathrm{NaOD}$, the exchange of deuterium was found to occur not only on the microfibril surface but also in the crystalline core (Wada et al. 1997). Despite prolonged exposure of the specimens to air, rehydrogenation occurred only on the surface, while the core remained deuterated.

In this report, we show an FTIR method to measure the accessibility and the size of cellulose microfibrils. This method is similar to the conventional deuteration technique for measuring the accessibility of cellulosic materials; however, the difference in our method is that the hydroxyl groups $\mathrm{O} 2 \mathrm{H}$, $\mathrm{O} 3 \mathrm{H}$, and $\mathrm{O} 6 \mathrm{H}$ in the crystalline region were initially completely deuterated. The sample was then rehydrogenated by soaking in water at $25{ }^{\circ} \mathrm{C}$, so that the OD moieties of the crystal surface were rehydrogenated. The ratio of $\mathrm{OH}$ to $\mathrm{OD}$ absorbance was used to calculate the number of surface vs. core cellulose chains in a microfibril. We applied our novel method to Valonia cellulose, whose microfibril have used as a standard sample because of the largest dimensions and high crystallinity.

Hydrogen recovery was considered as a measure of the penetration of hydrogen into the crystal, and it was estimated from the change in $\mathrm{OH}$ - and OD-stretching bands. The ratio of absorbance $(A)$ at the $\mathrm{OH}$ and $\mathrm{OD}$ regions, which are normalized by the absorbance at the $\mathrm{CH}$ and $\mathrm{CH}_{2}$ regions, respectively represents the OD remaining in a sample; $R_{\exp }$ (the experimental ratio) is obtained by the following equation:

$\mathrm{R}_{\exp }=A_{O D} /\left(A_{O H}+A_{O D}\right)$

Cellulose microfibrils consist of numerous linked glucose molecules, each of which exposes 3 hydroxyl groups. It is generally accepted that the cellulose structure has 2 types of $\mathrm{H}$ bonds: bonds formed between atoms of the same chain, and $\mathrm{H}$-bonds connecting neighboring chains in the same sheet. $\mathrm{O}-2-\mathrm{H}^{\cdots} \mathrm{O}-6$ and $\mathrm{O}-3-\mathrm{H} \cdots \mathrm{O}-5$ links exhibit intrachain $\mathrm{H}-b o n d s$, while the $\mathrm{O}-3-\mathrm{H} \cdots \mathrm{O}-6^{\prime}$ connections exhibit interchain H-bonds (Nishiyama et al. 2002, 2003). Moreover, $\mathrm{O}-3-\mathrm{H} \cdots \mathrm{O}-5$ bonds were found to be the most stable $\mathrm{H}$-bonds in cellulose. The adjacent oxygen $\mathrm{O}-2^{\prime}$ in $\mathrm{O}-6-\mathrm{H}^{\cdots} \mathrm{O}-3^{\prime}$ interchain $\mathrm{H}$ bonds can also serve as an acceptor. All the IR bands in the $3600-3000 \mathrm{~cm}^{-1}$ range are $\mathrm{O}-\mathrm{H}$ stretching mode in alcoholic groups. It is generally accepted that the sharpest band, i.e., that at $3343 \mathrm{~cm}^{-1}$, is assigned to the intrachain H-bond $\mathrm{O}-3-\mathrm{H} \cdots \mathrm{O}-5$ (Márechal and Chanzy 2000), and that at $2484 \mathrm{~cm}^{-1}$ is likely to be an O-3-D $\cdots \mathrm{O}-5$ bond. The $\mathrm{R}_{\exp }$ value was estimated based on the peaks of these bonds.

As a working model in this study, a microfibril consisting of $p \times q$ chains in a section was considered for simplicity (Figure 1). When deuterated microfibrils are washed in water at $25^{\circ} \mathrm{C}$, only half of the surface ODs is accessible to $\mathrm{OH}$ since the other half is buried in the core of the crystalline domain (Figure 1, hatched region). The number of surface chains is $2(p-1)+2(q-1)$, and 2 cellulose chains at the 4 corners are accessible to both sides; therefore, the $\mathrm{R}_{\text {calc }}$ (the calculated ratio) value at $25^{\circ} \mathrm{C}$ is given by the following equation: 


$$
\begin{aligned}
\mathrm{R}_{\text {calc }} & =\{(p-2) \times(q-2)+(p-1)+(q-1)-1\} / p \times q \\
& =(p-1) \times(q-1) / p \times q
\end{aligned}
$$

Assuming that $p=33$ and $q=38$ (Sugiyama et al. 1984) in the cellulose obtained from $V$. macrophysa, the $\mathrm{R}_{\text {calc }}$ value was estimated to be 0.943 , while the $\mathrm{R}_{\exp }$ value was 0.934 obtained for the sample washed at $25^{\circ} \mathrm{C}$. This small difference $(0.943$ vs. 0.934$)$ may be simply because of probable errors of measurements or of more disordered accessible regions in actual sample compared to simple estimation from the surface of cellulose microfibrils. Among organisms that synthesize cellulose, $V$. ventricosa synthesizes cellulose with one of the highest crystallinities; therefore, the microfibrils are closest to the surface/core model assumed for the present formulation, and thus the results agreed quite well.

We devised a stable and reproducible experimental method for accessibility measurement. Therefore, this technique can be applied to a variety of cellulose microfibrils produced by other organisms.

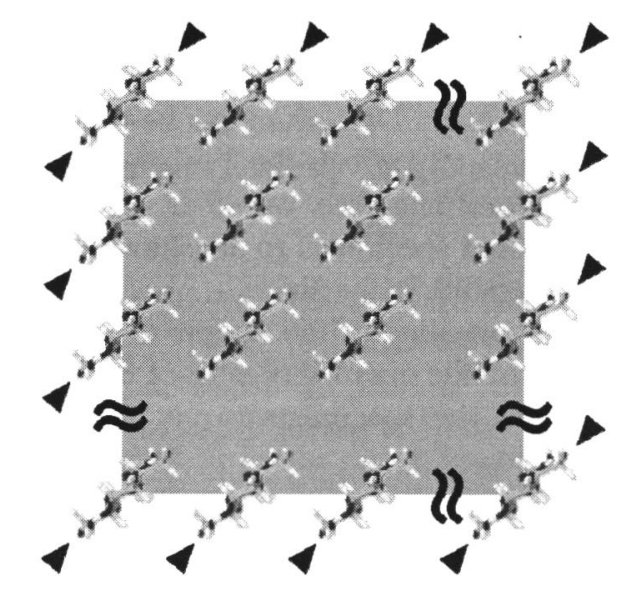

Figure 1 Schematic representation of the cellulose microfibril surface exposed to water at $25{ }^{\circ} \mathrm{C}$ after deuteration. The arrowhead indicates the parts of the cellulose chain that are accessible to $\mathrm{H}$.

\section{REFERENCES}

[1] Frilette V. J., Hanle J. and Mark H. (1948) Rate of exchange of cellulose with heavy water. J. Am. Chem. Soc. 70: 1107-1113.

[2] Márechal Y. and Chanzy H. (2000) The hydrogen bond network in I-beta cellulose as observed by infrared spectrometry. J. Mol. Struc. 523: 183-196.

[3] Nishiyama Y., Langan P. and Chanzy H. (2002) Crystal structure and hydrogen-bonding system in cellulose $\mathrm{I}_{\beta}$ from synchrotron X-ray and neutron fiber diffraction. J. Am. Chem. Soc. 124: 9074-9082.

[4] Nishiyama Y., Sugiyama J., Chanzy H. and Langan P. (2003) Crystal structure and hydrogen bonding system in cellulose $I_{\alpha}$, from synchrotron X-ray and neutron fiber diffraction. J. Am. Chem. Soc. 125: 14300-14306.

[5] Sugiyama J., Harada H. Fujiyoshi Y. and Uyeda N. 1984. High resolution observations of cellulose microfibrils. Mokuzai Gakkaishi 30: 98-99.

[6] Wada M., Okano T. and Sugiyama J. 1997. Synchrotron-radiated X-ray and neutron diffraction study of native cellulose. Cellulose 4: 221-232. 